\title{
Direct Measurement of the Density Matrix of a Quantum System
}

\author{
G. S. Thekkadath, L. Giner, Y. Chalich, M. J. Horton, J. Banker, and J. S. Lundeen \\ Department of Physics and Max Planck Centre for Extreme and Quantum Photonics, University of Ottawa, \\ 25 Templeton Street, Ottawa, Ontario K1N 6N5, Canada \\ (Received 26 April 2016; revised manuscript received 14 June 2016; published 12 September 2016)
}

\begin{abstract}
One drawback of conventional quantum state tomography is that it does not readily provide access to single density matrix elements since it requires a global reconstruction. Here, we experimentally demonstrate a scheme that can be used to directly measure individual density matrix elements of general quantum states. The scheme relies on measuring a sequence of three observables, each complementary to the last. The first two measurements are made weak to minimize the disturbance they cause to the state, while the final measurement is strong. We perform this joint measurement on polarized photons in pure and mixed states to directly measure their density matrix. The weak measurements are achieved using two walk-off crystals, each inducing a polarization-dependent spatial shift that couples the spatial and polarization degrees of freedom of the photons. This direct measurement method provides an operational meaning to the density matrix and promises to be especially useful for large dimensional states.
\end{abstract}

DOI: 10.1103/PhysRevLett.117.120401

Shortly after the inception of the quantum state, Pauli questioned its measurability, and in particular, whether or not a wave function can be obtained from position and momentum measurements [1]. This question, now referred to as the Pauli problem, draws on concepts such as complementarity and measurement in an attempt to demystify the physical significance of the quantum state. Indeed, the task of determining a quantum state is a central issue in quantum physics due to both its foundational and its practical implications. For instance, a method to verify the production of complicated states is desirable in quantum information and quantum metrology applications. Moreover, since a state fully characterizes a system, any possible measurement outcome can be predicted once the state is determined.

A wave function describes a quantum system that can be isolated from its environment, meaning the two are noninteracting and the system is in a pure state. More generally, open quantum systems can interact with their environment and the two can become entangled. In such cases, or even in the presence of classical noise, the system is in a statistical mixture of states (i.e., a mixed state), and one requires a density matrix to fully describe the quantum system. In fact, some regard the density matrix as more fundamental than the wave function because of its generality and its relationship to classical measurement theory [2].

The standard way of measuring the density matrix is by using quantum state tomography (QST). In QST, one performs an often overcomplete set of measurements in incompatible bases on identically prepared copies of the state. Then one fits a candidate state to the measurement results with the help of a reconstruction algorithm [3]. Many efforts have been made to optimize QST [4-7], but the scalability of the experimental apparatus and the complexity of the reconstruction algorithm renders the task increasingly difficult for large dimensional systems. In addition, since QST requires a global reconstruction, it does not provide direct access to coherences (i.e., off-diagonal elements), which are of particular interest in quantum physics.

Some recent work has focused on developing a direct approach to measuring quantum states [8-19]. Defining features of direct methods are that they can determine the state without complicated computations, and they can do so locally, i.e., at the location of the measurement probe. For example, direct measurement of the wave function has been achieved by performing a sequence consisting of a weak and a strong measurement of complementary variables (e.g., position and momentum) [8]. In the subensemble of trials for which the strong measurement results in a particular outcome (i.e., "postselection"), the average weak measurement outcome is a complex number known as the weak value $[20,21]$. The weak value is a concept that has proven to be useful in addressing fundamental questions in quantum physics [22-31], even beyond optics [32]. By foregoing postselection, previous work $[10,11]$ generalized the direct wave function measurement scheme to measure mixed quantum states. However, their method still does not provide direct access to individual density matrix elements. Reference [9] proposes a way to do this by performing an additional complementary measurement after the wave function measurement sequence: The second measurement serves as a phase reference and enables the first and last measurements to probe the coherence between any two chosen states in a certain basis. On top of its applications, a direct measurement method provides an operational meaning to the density matrix in terms of a sequence of three complementary measurements.

In this Letter, we experimentally demonstrate the method proposed in Ref. [9] by directly measuring any chosen 
element of a density matrix $\boldsymbol{\rho}_{\mathcal{S}}$ of a system $\mathcal{S}$. By repeating this for each element, we then measure the entire density matrix, thereby completely determining the state of the system. At the center of the method is a sequence of incompatible measurements $[33,34]$. In order for these measurements not to disrupt each other, they are made weak, a concept that we outline now (for a review, see Ref. [35]). Suppose one wishes to measure the observable $\boldsymbol{C}$. In von Neumann's model of measurement, the measured system $\mathcal{S}$ is coupled to a separate "pointer" system $\mathcal{P}$ whose wave function is initially centered at some position and has a width $\sigma$. This coupling proportionally shifts the position of the pointer by the value of $\boldsymbol{C}$ as described by the unitary translation $\boldsymbol{U}=\exp (-i \delta \boldsymbol{C p} / \hbar)$, where $\boldsymbol{p}$ is the pointer momentum operator and $\delta$ is the strength of the interaction. After the coupling, the pointer position $q$ is measured. On a trial by trial basis, if $\delta \gg \sigma$, the pointer position will be shifted by $\Delta q \approx \delta c$ and thus will indicate that the result of the measurement of $\boldsymbol{C}$ is $c$.

In contrast, in weak measurement $\delta \ll \sigma$, and the measurement result is ambiguous since it falls within the original position distribution of the pointer. However, this does have a benefit: The small interaction leaves the measured system relatively undisturbed and thus it can subsequently be measured again [36]. By repeating the weak measurement on an ensemble of systems and averaging, the shift of the pointer can be found unambiguously. This average shift is called the "weak average" $\langle\boldsymbol{C}\rangle_{\mathcal{S}}$ and is equal to the expectation value of a conventional (i.e., "strong") measurement: $\langle\boldsymbol{C}\rangle_{\mathcal{S}}=\operatorname{Tr}_{\mathcal{S}}\left[\boldsymbol{C} \boldsymbol{\rho}_{\mathcal{S}}\right]$ [9]. This differs from the weak value normally encountered in that there is no postselection.

Unlike in strong measurement, $\boldsymbol{C}$ can be non-Hermitian. This is the case when $\boldsymbol{C}$ is the product of incompatible observables (which normally disturb each other). Consequently, it is possible for the weak average to be complex. What does this imply? Both the position $\boldsymbol{q}$ and the momentum $\boldsymbol{p}$ of the pointer will be shifted according to $\langle\boldsymbol{C}\rangle_{\mathcal{S}}=(1 / \delta)\langle\mathbf{a}\rangle_{\mathcal{P}}$, where $\mathbf{a}=\boldsymbol{q}+i 2 \sigma^{2} \boldsymbol{p} / \hbar$ is the standard harmonic oscillator lowering operator scaled by $2 \sigma$ [37]. The real part and the imaginary part of the weak average are proportional to the average shift of the pointer's position and momentum, respectively.

Consider the weak measurement of an observable composed of the following three incompatible projectors:

$$
\Pi_{a_{i} a_{j}}=\boldsymbol{\pi}_{a_{j}} \boldsymbol{\pi}_{b_{0}} \boldsymbol{\pi}_{a_{i}},
$$

where $\pi_{a_{i}}=\left|a_{i}\right\rangle\left\langle a_{i}\right|$ and $\boldsymbol{\pi}_{b_{0}}=\left|b_{0}\right\rangle\left\langle b_{0}\right|$, which are composed of eigenstates of the observables $\boldsymbol{A}$ and $\boldsymbol{B}$, respectively. These are maximally incompatible, or "complementary," in the sense that $\left|\left\langle a_{i} \mid b_{0}\right\rangle\right|=1 / \sqrt{d}$ for a $d$-dimensional Hilbert space. In the basis of the eigenstates of $\boldsymbol{A}$, a density matrix element is given by $\rho_{\mathcal{S}}(i, j)=\left\langle a_{i}\left|\boldsymbol{\rho}_{\mathcal{S}}\right| a_{j}\right\rangle$. This can be connected to the weak average of the measurement sequence in Eq. (1):

$$
\left\langle\Pi_{a_{i} a_{j}}\right\rangle_{\mathcal{S}}=\operatorname{Tr}_{\mathcal{S}}\left[\boldsymbol{\pi}_{a_{j}} \boldsymbol{\pi}_{b_{0}} \boldsymbol{\pi}_{a_{i}} \boldsymbol{\rho}_{\mathcal{S}}\right]=\rho_{\mathcal{S}}(i, j) / d
$$

In fact, one can replace the weak measurement of the last projector, $\boldsymbol{\pi}_{a_{j}}$, by a strong measurement without affecting the weak average [9], thereby reducing the complexity of the measurement apparatus. Thus, any density matrix element can be obtained by selecting the first and last projectors in the measurement sequence. Whichever state $\left|b_{0}\right\rangle$ that is chosen for the middle complementary projector serves as a reference for zero phase in the density matrix by fixing $\theta=0$ for all values of $a$ in $\left\langle a \mid b_{0}\right\rangle=\exp (i \theta) / \sqrt{d}$. As such, $\left|b_{0}\right\rangle$ should not be changed while measuring $\rho_{\mathcal{S}}$.

The experimental setup is shown in Fig. 1. We demonstrate the technique by directly measuring the density matrix of a photon polarization state. This is possibly the simplest system for a demonstration, but it is also an important one since it can act as a qubit from which larger and more complicated quantum states can be constructed, such as in quantum computing. A HeNe laser at $633 \mathrm{~nm}$ is sent through a polarizing beam splitter (PBS) to ensure that it is polarized. We treat the bright polarized beam as a source of a large number of identically prepared polarized photons. Instead of using a separate system, we use the $x$ and $y$ transverse spatial distributions of the photons as pointers. Both are Gaussian with widths $\sigma=$ $250 \mu \mathrm{m}(830 \mu \mathrm{m}$ FWHM $)$ that are set using a telescopic arrangement of two convex lenses $\left(f_{1}=50 \mathrm{~mm}\right.$ and
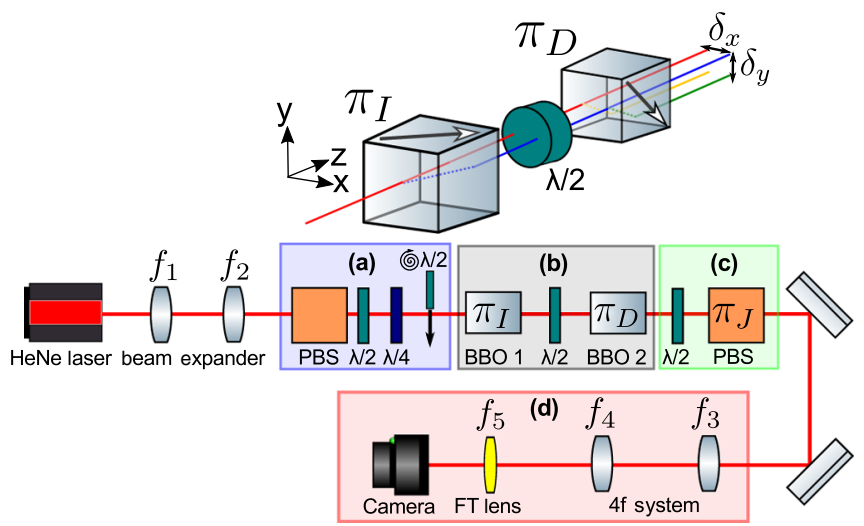

FIG. 1. Direct measurement experimental setup. (a) State preparation: We use a HeNe laser as a source of photons. The photon polarization state is set using a half-wave plate $(\lambda / 2)$ and a quarter-wave plate $(\lambda / 4)$. A spinning $\lambda / 2$ is included when generating mixed states. (b) Weak measurements: Two subsequent weak measurements, $\boldsymbol{\pi}_{I}$ and $\boldsymbol{\pi}_{D}$, are performed, each with a walk-off crystal (BBO) that couples the polarization to a spatial degree of freedom, $x$ or $y$, our measurement pointers. Note that $\delta_{x}=\delta_{y} \equiv \delta$. (c) Strong measurement: The final measurement $\boldsymbol{\pi}_{J}$ is performed by a polarizing beam splitter (PBS), and the projection direction $J$ is set by a $\lambda / 2$. (d) Imaging: A $4 f$ arrangement of lenses forms an image of the crystal plane onto a camera, allowing us to measure pointer positions. An additional Fourier transform (FT) lens, either spherical or cylindrical, is used to measure pointer momenta. 
$\left.f_{2}=100 \mathrm{~mm}\right)$. We set the photon polarization state $\boldsymbol{\rho}_{\mathcal{S}}$ using a half-wave plate $(\lambda / 2)$ and a quarter-wave plate $(\lambda / 4)$.

A weak measurement of polarization is implemented by coupling the polarization degree of freedom (our system) to a spatial one (a pointer). This is accomplished with a walkoff crystal [beta barium borate (BBO)] that shifts the $|I\rangle$ polarization component along $x$ by $\delta=176 \mu \mathrm{m}$. If $\delta \gg \sigma$ this implements a strong measurement of $\boldsymbol{\pi}_{I}=|I\rangle\langle I|$ since the photon position unambiguously determines $\pi_{I}$. If $\delta \ll \sigma$ this is a weak measurement of $\boldsymbol{\pi}_{I}$ [38]. In our demonstration, we find each of the four polarization density matrix elements, $\rho_{\mathcal{S}}(I, J)$, by measuring the three projector observable, $\boldsymbol{\pi}_{J} \boldsymbol{\pi}_{D} \boldsymbol{\pi}_{I}$, where either $I$ or $J$ can be horizontal $(H)$ or vertical $(V)$ polarization and $|D\rangle=(|H\rangle+|V\rangle) / \sqrt{2}$ is a complementary state, the diagonal polarization.

Coupling a joint observable $\boldsymbol{E} \boldsymbol{F}$ such as $\boldsymbol{\pi}_{D} \boldsymbol{\pi}_{I}$ to a single pointer is challenging for photons. Instead, we follow a strategy commonly used for joint strong measurements (e.g., those in Bell's inequalities) in which one independently measures single observables and then evaluates correlations between the independent results. In von Neumann's model, this corresponds to having two independent pointers so that $\langle\boldsymbol{E} \boldsymbol{F}\rangle_{\mathcal{S}}=(1 / \delta)^{2}\left\langle\boldsymbol{q}_{E} \boldsymbol{q}_{F}\right\rangle_{\mathcal{P}}$, where $\boldsymbol{q}_{m}$ is the position of the $m=E, F$ pointer. In the weak measurement analog, proposed in Ref. [37], one replaces $\boldsymbol{q}_{m}$ with $\mathbf{a}_{m}$, so $\langle\boldsymbol{E} \boldsymbol{F}\rangle_{\mathcal{S}}=(1 / \delta)^{2}\left\langle\mathbf{a}_{E} \mathbf{a}_{F}\right\rangle_{\mathcal{P}}[9,39]$. Thus, we can couple $\boldsymbol{\pi}_{I}$ and $\boldsymbol{\pi}_{D}$ to separate pointers and then measure correlations between the momenta and positions of these pointers to find the weak average. The final measurement in the sequence $\boldsymbol{\pi}_{J}$ is strong, so the full joint expectation value is

$$
\left\langle\Pi_{I J}\right\rangle_{\mathcal{S}}=\left(\frac{1}{\delta}\right)^{2} \operatorname{Tr}_{\mathcal{T}}\left[\boldsymbol{\pi}_{J} \mathbf{a}_{D} \mathbf{a}_{I} \boldsymbol{\rho}_{\mathcal{T}}\right]=\rho_{\mathcal{S}}(I, J) / 2,
$$

where $\mathcal{T}=\mathcal{S} \otimes \mathcal{P}$ indicates the total Hilbert space, combining the pointers and the system $(d=2)$.

In our experiment, we conduct two independent weak measurements by sequentially introducing two walk-off crystals in the beam path (see the Supplemental Material [40] for the alignment procedure). The first measures $\pi_{I}$ by inducing a displacement $\delta$ along $x$. Combined with a $\lambda / 2$ at $22.5^{\circ}$, the second crystal induces a displacement $\delta$ along $y$, measuring $\boldsymbol{\pi}_{D}$. The last projector $\boldsymbol{\pi}_{J}$, the strong measurement, is implemented by a second $\lambda / 2$ and a PBS where the $\lambda / 2$ is used to choose the projected state $J=H, V$, i.e., a $J$ polarizer.

The lowering operators in the total pointer-system expectation value in Eq. (3) imply the measurement of positions and momenta of the photons. Experimentally, we measure quantities such as the probability that a photon is transmitted through the final $J$ polarizer and also has horizontal position $x$ and vertical position $y$, i.e., $\operatorname{Prob}(x, y, J)$ [41]. From this, we can find expectation values such as $\iint x y \operatorname{Prob}(x, y, J) d x d y \equiv\langle\boldsymbol{x} \boldsymbol{y}\rangle_{\mathcal{P}, J}$ (see the
Supplemental Material [40] for an example). Then the density matrix elements can be directly related to the joint position $(\boldsymbol{x}, \boldsymbol{y})$ and momentum $\left(\boldsymbol{p}_{x}, \boldsymbol{p}_{y}\right)$ expectation values of the pointer state:

$$
\begin{aligned}
& \operatorname{Re}\left[\rho_{\mathcal{S}}(I, J)\right]=\frac{2}{\delta^{2}}\left(\left\langle\boldsymbol{x}_{I} \boldsymbol{y}_{D}\right\rangle_{\mathcal{P}, J}-\frac{\sigma^{2}}{\sigma_{p}^{2}}\left\langle\boldsymbol{p}_{x I} \boldsymbol{p}_{y D}\right\rangle_{\mathcal{P}, J}\right), \\
& \operatorname{Im}\left[\rho_{\mathcal{S}}(I, J)\right]=\frac{2}{\delta^{2}} \frac{\sigma}{\sigma_{p}}\left(\left\langle\boldsymbol{p}_{x I} \boldsymbol{y}_{D}\right\rangle_{\mathcal{P}, J}+\left\langle\boldsymbol{x}_{I} \boldsymbol{p}_{y D}\right\rangle_{\mathcal{P}, J}\right) .
\end{aligned}
$$

Equation (4) is expressed using $\sigma$ and $\sigma_{p}$, where $\sigma \sigma_{p}=\hbar / 2$, to explicitly remove the unit dependence of position and momentum. The subscript $I$ in, e.g., $\left\langle\boldsymbol{x}_{I} \boldsymbol{y}_{D}\right\rangle$ indicates that the projector $\boldsymbol{\pi}_{I}$ is coupled to the $\boldsymbol{x}$ pointer.

We measure the four joint expectation values in Eq. (4) one at a time using a camera (a CMOS sensor with resolution $2560 \times 1920$ and a pixel side length of $2.2 \mu \mathrm{m})$. The position expectation value $\langle\boldsymbol{x} \boldsymbol{y}\rangle$ of the pointer state is obtained using two convex lenses $\left(f_{3}=1000 \mathrm{~mm}\right.$ and $f_{4}=1200 \mathrm{~mm}$ ) in a $4 f$ arrangement that images the crystal plane onto the camera. The momentum expectation value $\left\langle\boldsymbol{p}_{x} \boldsymbol{p}_{y}\right\rangle$ is obtained by adding a spherical lens $\left(f_{5}=1000 \mathrm{~mm}\right)$ one focal length from the camera. We replace the spherical lens with a cylindrical one (also $f_{5}$ ) to take a one-dimensional Fourier transform of the pointer states and measure the expectation values $\left\langle\boldsymbol{p}_{x} \boldsymbol{y}\right\rangle$ and $\left\langle\boldsymbol{p}_{y} \boldsymbol{x}\right\rangle$ by rotating the axis cylindrical lens. In order to obtain each and every density matrix element, we repeat these four measurements for all combinations of $(I, J)$.

First, we measure the density matrix elements of the pure state $|\psi\rangle=\cos \theta|H\rangle-e^{i \alpha \pi / 2} \sin \theta|V\rangle$ :

$\boldsymbol{\rho}=|\psi\rangle\langle\psi|=\left(\begin{array}{cc}\cos ^{2} \theta & -e^{-i \alpha \pi / 2} \cos \theta \sin \theta \\ -e^{i \alpha \pi / 2} \cos \theta \sin \theta & \sin ^{2} \theta\end{array}\right)$.

Figure 2(a) shows density matrix elements along path 1 in the Poincare sphere, which is traced by setting $\alpha=0$ (i.e., removing the $\lambda / 4$ ) and varying the fast axis of the $\lambda / 2$ such that $\theta \in\left[0,180^{\circ}\right]$. Figure 2(b) shows the same density matrix elements along path 2 , which is by traced by setting $\alpha=-1$ (i.e., the $\lambda / 4$ fast axis is fixed) and again varying the fast axis of the $\lambda / 2$ such that $\theta \in\left[0,180^{\circ}\right]$. As can be seen, the measured density matrix elements closely follow the theory curve. Deviations from the curve (e.g., near $\theta=90^{\circ}$ ) are likely due to imperfections in the wave plates, which can introduce systematic errors both when preparing the polarization state and aligning the $\mathrm{BBO}$ crystals.

Next, we generate mixed states by creating an incoherent combination of pure states. This is achieved by introducing a spinning $\lambda / 2$ in the preparation stage. This $\lambda / 2$ rotates sufficiently fast such that, over the exposure time of the camera, the measured result contains contributions from many polarization states [40]. Specifically, we produce 


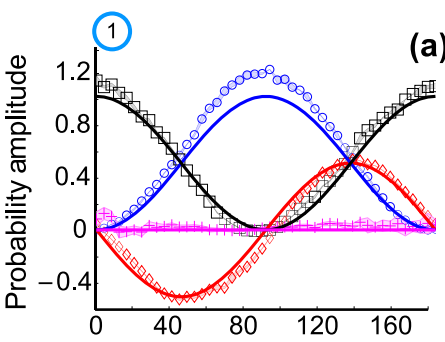

(a)

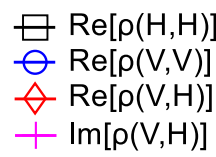

(2) Input state $\theta$ (deg)
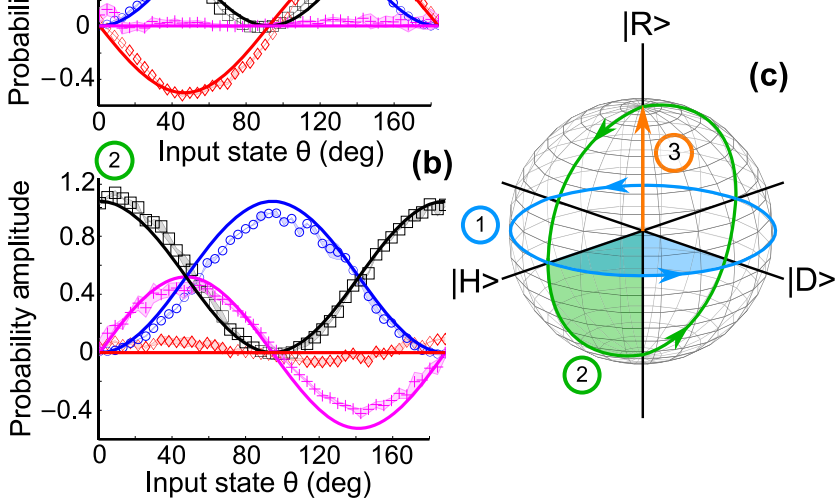

FIG. 2. Direct measurement of density matrix elements for pure polarization states. (a) and (b) Density matrix elements along paths 1 and 2 in the Poincaré sphere, respectively. The bold lines are the theoretical matrix elements given by Eq. (5), while the markers are data points. The shaded region in these plots represents one standard deviation from averaging over ten trials and is mostly smaller than the size of the markers. (c) Poincare sphere: Path 3 corresponds to the measurement of mixed states, shown in Fig. 3. The shaded regions indicate an interval of $\Delta \theta=$ $45^{\circ}$ to help the reader link the paths to the $\theta$ axes in (a) and (b).

$$
\boldsymbol{\rho}=\left(\begin{array}{cc}
1 / 2 & i \sin \phi \cos \phi \\
-i \sin \phi \cos \phi & 1 / 2
\end{array}\right)
$$

where $\phi$ is the angle between horizontal and the fast axis of the $\lambda / 4$. We generate a series of such mixed states [see
Fig. 3(b)] and vary their purity $\operatorname{Tr}\left[\rho^{2}\right]$ between $1 / 2$ and 1 by adjusting $\phi$. This corresponds to path 3 in the Poincaré sphere, as shown in Fig. 2(c). To measure the accuracy of our measured density matrices, we compute the trace distance $\left|\operatorname{Tr}\left[\sqrt{(\boldsymbol{\beta}-\boldsymbol{\rho})^{\dagger}(\boldsymbol{\beta}-\boldsymbol{\rho})}\right]\right| / 2(\boldsymbol{\beta}$ is the measured state), which is shown in Fig. 3(c). The trace distance can be interpreted as a measure of the maximum probability of distinguishing between two states, $\boldsymbol{\rho}$ and $\boldsymbol{\beta}$, with an optimal measurement. For our results, this probability is always less than $4.9 \%$. We also note that the measured density matrix may not be positive semidefinite due to measurement uncertainties. Consequently, if one requires a positive semidefinite matrix, one would need to employ additional algorithms such as a maximum-likelihood estimation.

To summarize, we directly measure the density matrix elements of photons in both pure and mixed polarization states using three sequential measurements, each complementary to the last. The first two measurements are weak to minimize their disturbance on the state, while the last measurement is strong. The average joint result of this measurement sequence gives any chosen density matrix element, and hence it can be used to operationally define the density matrix.

We anticipate that this method will be of use in practical applications. Since the last measurement can be weak, it could function as a noninvasive probe to determine a quantum state in situ, such as during a quantum computation or a molecular evolution. Moreover, one could envisage directly observing global properties of a state, such as the existence of nonclassical correlations [34], by measuring coherences or entanglement witnesses with our method. Lastly, direct measurement has already proven to be efficient for measuring large dimensional pure states in

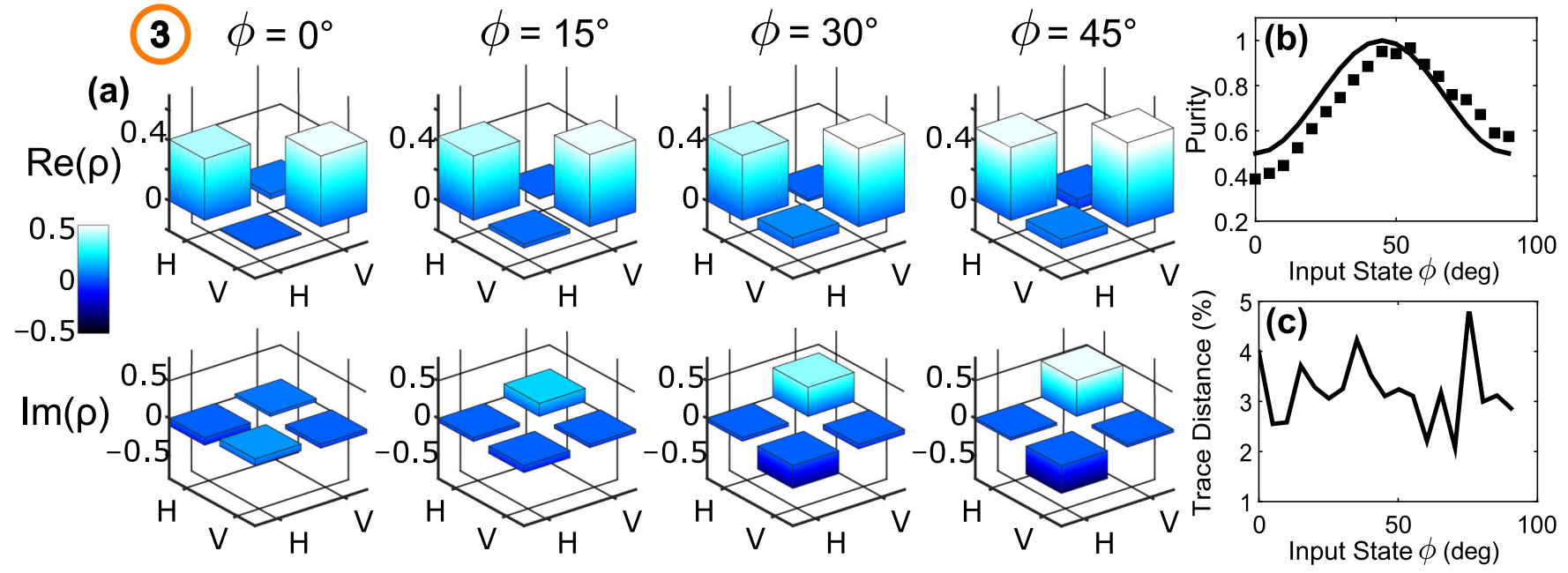

FIG. 3. Direct measurement of mixed states. (a) Measured density matrices. The color is proportional to the measured probability amplitudes. (b) States with various degrees of purity $\operatorname{Tr}\left[\rho^{2}\right]$ can be generated by varying the fast axis angle $\phi$ of a $\lambda / 4$, as shown in Eq. (6). The bold line is the theory, while the markers are data points. The states follow path 3 in the Poincaré sphere shown in Fig. 2. We do not show statistical uncertainties, as they are smaller than the markers. (c) The trace distance is half the Euclidean distance between the measured and theory states on the Poincaré sphere, and it is always less than 0.049 (i.e., 4.9\%). 
various physical systems $[13,17,18]$. Quantum state tomography typically requires $O\left(d^{2}\right)$ measurements in $O(d)$ bases and finds the full density matrix at once. Thus, as $d$ increases, the experimental procedure and the reconstruction algorithm become increasingly complicated. In contrast, our direct measurement method requires three measurements in only two bases to determine any chosen density matrix element regardless of the system dimension $d$. Consequently, in systems with a large $d$, the method is an attractive alternative to tomography as a way to locally characterize a potentially mixed quantum state.

This work was supported by the Canada Research Chairs (CRC) Program, the Natural Sciences and Engineering Research Council (NSERC), and the Cananda Excellence Research Chairs (CERC) Program.

[1] W. Pauli, General Principles of Quantum Mechanics (Springer, Berlin, 1980), p. 67.

[2] L. Hardy, arXiv:quant-ph/0101012.

[3] Some reconstruction algorithms restrictively fit the measurement results to reconstruct only physical (i.e., positive semidefinite and normalized) states.

[4] W. K. Wootters and B. D. Fields, Ann. Phys. (N.Y.) 191, 363 (1989).

[5] J. M. Renes, R. Blume-Kohout, A. J. Scott, and C. M. Caves, J. Math. Phys. (N.Y.) 45, 2171 (2004).

[6] T. Durt, B.-G. Englert, I. Bengtsson, and K. Życzkowski, Int. J. Quantum. Inform. 08, 535 (2010).

[7] N. Bent, H. Qassim, A. A. Tahir, D. Sych, G. Leuchs, L. L. Sánchez-Soto, E. Karimi, and R. W. Boyd, Phys. Rev. X 5, 041006 (2015).

[8] J. S. Lundeen, B. Sutherland, A. Patel, C. Stewart, and C. Bamber, Nature (London) 474, 188 (2011).

[9] J. S. Lundeen and C. Bamber, Phys. Rev. Lett. 108, 070402 (2012).

[10] C. Bamber and J. S. Lundeen, Phys. Rev. Lett. 112, 070405 (2014).

[11] J. Z. Salvail, M. Agnew, A. S. Johnson, E. Bolduc, J. Leach, and R. W. Boyd, Nat. Photonics 7, 316 (2013).

[12] M. Mirhosseini, O. S. Magaña-Loaiza, S. M. Hashemi Rafsanjani, and R.W. Boyd, Phys. Rev. Lett. 113, 090402 (2014).

[13] M. Malik, M. Mirhosseini, M. P. J. Lavery, J. Leach, M. J. Padgett, and R. W. Boyd, Nat. Commun. 5, 3115 (2014).

[14] J. Fischbach and M. Freyberger, Phys. Rev. A 86, 052110 (2012).

[15] A. Di Lorenzo, Phys. Rev. Lett. 110, 010404 (2013).

[16] S. Wu, Sci. Rep. 3, 1193 (2013).

[17] E. Bolduc, G. Gariepy, and J. Leach, Nat. Commun. 7, 10439 (2016).

[18] Z. Shi, M. Mirhosseini, J. Margiewicz, M. Malik, F. Rivera, Z. Zhu, and R. W. Boyd, Optica 2, 388 (2015).
[19] G. Vallone and D. Dequal, Phys. Rev. Lett. 116, 040502 (2016).

[20] Y. Aharonov, D. Z. Albert, and L. Vaidman, Phys. Rev. Lett. 60, 1351 (1988).

[21] Y. Aharonov and L. Vaidman, Phys. Rev. A 41, 11 (1990).

[22] K. J. Resch, J. S. Lundeen, and A. M. Steinberg, Phys. Lett. A 324, 125 (2004).

[23] J. S. Lundeen and A. M. Steinberg, Phys. Rev. Lett. 102, 020404 (2009).

[24] K. Yokota, T. Yamamoto, M. Koashi, and N. Imoto, New J. Phys. 11, 033011 (2009).

[25] A. Palacios-Laloy, F. Mallet, F. Nguyen, P. Bertet, D. Vion, D. Esteve, and A. N. Korotkov, Nat. Phys. 6, 442 (2010).

[26] M. E. Goggin, M. P. Almeida, M. Barbieri, B. P. Lanyon, J. L. O'Brien, A. G. White, and G. J. Pryde, Proc. Natl. Acad. Sci. U.S.A. 108, 1256 (2011).

[27] J. Dressel, C. J. Broadbent, J. C. Howell, and A. N. Jordan, Phys. Rev. Lett. 106, 040402 (2011).

[28] A. M. Steinberg, Phys. Rev. Lett. 74, 2405 (1995).

[29] S. Kocsis, B. Braverman, S. Ravets, M. J. Stevens, R. P. Mirin, L. K. Shalm, and A. M. Steinberg, Science 332, 1170 (2011).

[30] H. M. Wiseman, Phys. Rev. A 65, 032111 (2002).

[31] F. Piacentini, A. Avella, M. P. Levi, R. Lussana, F. Villa, A. Tosi, F. Zappa, M. Gramegna, G. Brida, I. P. Degiovanni, and M. Genovese, Phys. Rev. Lett. 116, 180401 (2016).

[32] I. Shomroni, O. Bechler, S. Rosenblum, and B. Dayan, Phys. Rev. Lett. 111, 023604 (2013).

[33] F. Piacentini, M. P. Levi, A. Avella, E. Cohen, R. Lussana, F. Villa, A. Tosi, F. Zappa, M. Gramegna, G. Brida, I. P. Degiovanni, and M. Genovese, arXiv:1508.03220.

[34] Y. Suzuki, M. Iinuma, and H.F. Hofmann, arXiv:1606.00148.

[35] J. Dressel, M. Malik, F. M. Miatto, A. N. Jordan, and R. W. Boyd, Rev. Mod. Phys. 86, 307 (2014).

[36] A. C. Ipsen, Phys. Rev. A 91, 062120 (2015).

[37] J. S. Lundeen and K. J. Resch, Phys. Lett. A 334, 337 (2005).

[38] A walk-off crystal is used for a weak measurement of polarization in, for example, Refs. [11] and [42].

[39] G. Mitchison, Phys. Rev. A 77, 052102 (2008).

[40] See Supplemental Material at http://link.aps.org/ supplemental/10.1103/PhysRevLett.117.120401 for information on the BBO alignment procedure, the mixed state generation, and, an example of a pointer state expectation value calculation.

[41] We note that this is not the same as a postselection since we do not renormalize the state after passing through the $J$ polarizer; i.e., the probability is not conditional on $\boldsymbol{\pi}_{J}$. $\operatorname{Prob}(x, y, J)$ is one value of the total normalized probability distribution.

[42] N. W. M. Ritchie, J. G. Story, and R. G. Hulet, Phys. Rev. Lett. 66, 1107 (1991). 